\title{
Development, Characterization and Evaluation of Tinidazole Nanosuspension for Treatment of Amoebiasis
}

Pawar RN, Chavan SN* and Menon MD

Department of Pharmaceutics, Bombay College of Pharmacy, Mumbai, Maharashtra, India

\begin{abstract}
Amoebiasis is an infectious disease of gastrointestinal tract caused by the protozoan, Entamoeba histolytica. This disease is widely distributed worldwide and is endemic in most parts of India and other developing countries. The protozoa multiply rapidly in their hosts. Since effective vaccines are as yet unavailable, chemotherapy is the major strategy to treat infected individuals and reduce transmission. Nitroimmidazoles, like Tinidazole (TNZ) form an important class of drugs, which are active against both intestinal and systemic forms of amoebiasis. Most of these drugs have high dose and metallic to bitter taste which result in poor patient compliance. In the present study, nanosuspension was developed by nanoprecipitation method for oral delivery using Tinidazole as model drugs with improved its dissolution rate and hence bioavailability. With improved bioavailability it may be possible to reduce the dose requirement, which would be especially advantageous for paediatric patients. Ex-vivo absorption studies performed using the rat intestinal model showed an enhanced absorption from the nanosuspension as compared to micronized drug.
\end{abstract}

Keywords: Nanosuspension; Bioavailability; Nanoprecipitation; Taste masking; Amoebiasis

\section{Introduction}

Solubility is important factor for drug efficacy, independent of route of administration, especially when drug is poorly soluble in both aqueous as well as organic media. This is a major challenge before pharmaceutical companies while developing new pharmaceutical products as very low amount of active substance is either insoluble or poorly soluble in water. Oral administration of such low soluble drugs in humans and laboratory animals frequently leads to incomplete and irregular absorption from the gastrointestinal tract, since the dissolution of the active substance is the major rate limiting step in the absorption process. Enhancement of oral bioavailability by micronization is not sufficient for many of the poorly soluble new chemical entities (NCE), the next step is Nanosization [1].

Some of the methods investigated as potential oral novel systems for poorly and sparingly soluble drugs include microparticles, nanoparticles, solid dispersions, microemulsions etc. Nanosuspensions are defined as ultra-fine suspensions of pure drug nanoparticles stabilized with a surfactant or surfactant mixtures. The poorly soluble and oil soluble compounds can be formulated as nanosuspensions also existing drugs can be reformulated to eliminate the toxicologically less favorable excipients. Such nanosized particles have high crystal energy, indicated by a high melting point, which reduces the tendency of the crystal to dissolve, regardless of the solvent. Different hydrophobic drugs have already been formulated successfully in this way, eg: Naproxen, Clofazimine, Nimesulide, Omeprazole, Mitotane, and Nifedipine [1].

An important advantage of nanosuspensions is the increase in saturation solubility, hence the dissolution rate of compound. This increase in the dissolution rate is may be due to increased surface area of particles. Another important feature of nanosuspensions is altered crystalline structure (increase in the amorphous fraction or may be creating totally amorphous particles) [1]. The drug candidates with poor solubility and dissolution-rate limited absorption generally exhibits low and variable oral bioavailability. Due to poor oral bioavailability such drug candidate would have to be administered in high doses thus making the therapy costly.
Atovaquone and Bupravaquone an orally administered antibiotics are the god examples of this type. Nanosizing of such drugs leads to a drastic increase in their oral absorption and consequently bioavailability. The increase in bioavailability is due to surface area (due to reduction in particle size by 337 fold), increased saturation solubility and increase in dissolution velocity. The drug dose reduction is possible with enhancement in bioavailability making therapy cost effective and demolishing excessive drug dumping in the body [2].

Nanosuspensions as drug delivery systems are advantageous since they have better physical stability due to small size of nanoparticles experiencing less gravitational force as compared to large microparticles, flocculation and ostwald ripening are also absent in case of nanoparticles [3-7].

In the recent studies nanoprecipitation method is widely used to increase solubility and bioavailability of the drug [8-16].

\section{Materials and Methods}

Tinidazole (gift sample from Ajanta Pharmaceuticals, Mumbai, India), Pluronic F127 and Cremophor-EL (BASF corporation, India), HPMC K4M (Colorcon Asia Private Ltd.), Soyalecithin Lecimuthin ${ }^{\circ}$ (Degussa), Tween 80, Lactose Anhydrous, Maltodextrin and Polyethylene Glycol-PEG 600 (S. D. Fine chemicals), Mannitol BP (Sarabhai M Chemicals) and Trehalose (Hyashibara, Japan). All other chemicals and reagents were of analytical grade.

\section{Preparation of nanosuspension}

Nanoprecipitation method was attempted, because of

*Corresponding author: Smita N. Chavan, Department of Pharmaceutics, Bombay College of Pharmacy, Mumbai, Maharashtra, India, Tel: 9820751344; E-mail:maladmbcp@yahoo.co.in

Received November 23, 2016; Accepted December 24, 2016; Published December 27, 2016

Citation: Pawar RN, Chavan SN, Menon MD (2016) Development, Characterization and Evaluation of Tinidazole Nanosuspension for Treatment of Amoebiasis. J Nanomed Nanotechnol 7: 413. doi: 10.4172/2157-7439.1000413

Copyright: $\odot 2016$ Pawar RN, et al. This is an open-access article distributed under the terms of the Creative Commons Attribution License, which permits unrestricted use, distribution, and reproduction in any medium, provided the original author and source are credited. 
Citation: Pawar RN, Chavan SN, Menon MD (2016) Development, Characterization and Evaluation of Tinidazole Nanosuspension for Treatment of Amoebiasis. J Nanomed Nanotechnol 7: 413. doi: 10.4172/2157-7439.1000413

the simplicity of the method, and feasibility to scale up. Several surfactants (Tween-80, Sodium Lauryl sulphate (SLS), Pluronic F-127, HPMC K4M, SLS, Cremophor-EL, Soyalecithin) and solvents (Dichloromethane (DCM), Ethyl acetate, Acetone) were screened to develop stable nanosuspension formulation. Of these soyalecithin and acetone were found best for TNZ nanosuspension (Table 1).

Nanosuspension was prepared by drop wise addition of organic phase (100 mg of TNZ in $25 \mathrm{ml}$ of acetone) to aqueous phase (50 $\mathrm{mg}$ of soyalecithin in $15 \mathrm{ml}$ distilled water) with continuous stirring. The emulsion thus formed was then subjected to evaporation at $50^{\circ} \mathrm{C}$ using Rotavapor under vacuum. After complete removal of acetone the preformed suspension was then diluted with remaining $5 \mathrm{ml}$ of filtered water to adjust final volume to $20 \mathrm{ml}$. To mask bitter taste and to improve patient compliance of the formulation different flavoring and coloring agents were evaluated. Selected final optimized batch of nanosupension was subjected to freeze-drying. Different cryoprotectants like maltodextrin, trehalose, lactose and mannitol were evaluated, amongst which trehalose was found to be the best.

\section{Physicochemical characterization}

The developed freeze dried formulation was characterized for drug content, particle size, $\mathrm{pH}$, residual solvent, ease of reconstitution, moisture content, in-vitro drug release, saturation soyalecithin, IR Spectroscopy, Differential Scanning Calorimetry (DSC), X-ray diffractometric analysis (XRD), Environment scanning electron microscopy (ESEM) and stability.

\section{Ex-vivo absorption studies}

The objective of ex-vivo absorption studies was to compare the rate and extent of absorption of the formulated nanosuspensions with that of micronized drug, marketed tablet using the rat intestinal model. Ex vivo absorption study was carried out using the selected freeze-dried sample of developed formulation, physical mixture of formulation, free drug (micronized TNZ) and its marketed tablet.

The inner lumen (villi portion) of freshly excised rat intestine tied at one end and through other end dispersed samples of TNZ were injected with help of a tuberculin syringe and then tied. These segments were then tied onto the paddle of dissolution USP apparatus Type II

\begin{tabular}{|l|c|c|c|c|} 
Solvent & Surfactant & $\begin{array}{c}\text { Drug: surfactant } \\
\text { Ratio }\end{array}$ & $\begin{array}{c}\text { Solvent } \\
\text { quantity }\end{array}$ & $\begin{array}{c}\text { Particle } \\
\text { Size (nm) }\end{array}$ \\
\hline Acetone & Soyalecithin & $1: 0.25$ & $93.75 \mathrm{ml}$ & 217.0 \\
\hline
\end{tabular}

Table 1: Final batch of nanosuspension prepared by nanoprecipitation method.
(Electro lab TDT $06 \mathrm{~T}$ ) and immersed in phosphate buffer ( $\mathrm{pH} 6.8$ ) medium. The study was performed at $50 \mathrm{rpm}$.

\section{Results and Discussion}

Nanosuspension with $0.75 \%$ TNZ, $0.19 \%$ soyalecithin showed a particle size of $217.0 \mathrm{~nm}$ and formed a homogenous suspension on reconstitution, with $7 \%$ and $10 \%$ increase in dissolution rate, compared to micronized drug and marketed tablet respectively. Within 2 hours $95 \%$ of drug was released from nanosuspension as compared to micronized drug (88\%) and marketed product (85\%). About $10 \%$ increase in saturation solubility was observed.

Taste masking of the formulation was successful with inclusion of $1.2 \%$ Peppermint water and $0.2 \%$ Sucralose. Ex-vivo absorption studies showed an enhanced absorption from the nanosuspension (94\%)

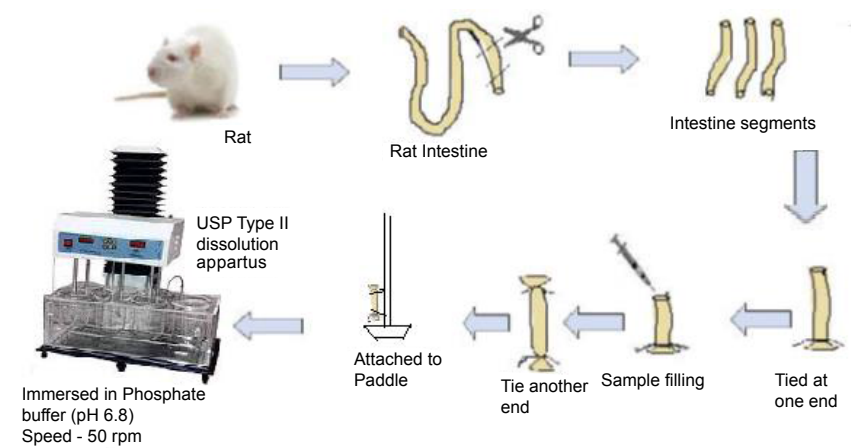

Figure 1: Procedure of Ex-vivo absorption studies.

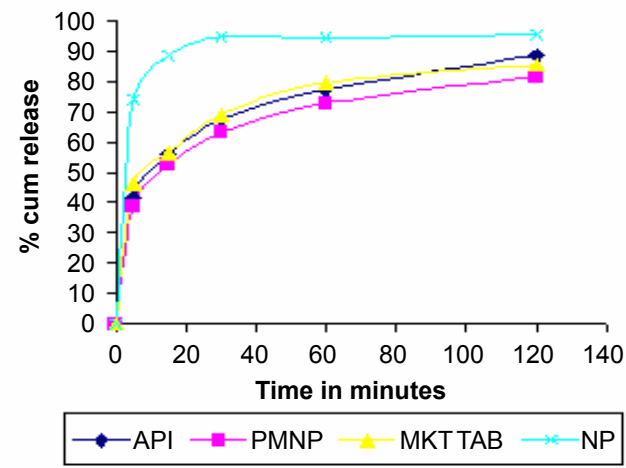

Figure 2: In-vitro drug release profile of nanosuspension.

\begin{tabular}{|c|c|c|}
\hline Characteristics & Before freeze drying & After freeze drying \\
\hline Mean Particle Size, polydispersity index & $\begin{array}{c}216.6 \mathrm{~nm} \pm 92.09 \mathrm{~nm} \\
(0.332)\end{array}$ & $\begin{array}{c}325.3 \mathrm{~nm} \pm 151.95 \mathrm{~nm} \\
(0.858)\end{array}$ \\
\hline Ease of reconstitution & Homogenous suspension & Homogenous suspension \\
\hline Drug Content $(\% w / w)^{\#}$ & $98.1 \pm 0.5$ & $97.9 \pm 0.5$ \\
\hline $\mathrm{pH}$ & $6.4 \pm 0.08$ & $6.39 \pm 0.05$ \\
\hline FTIR spectroscopy & - & No interaction between drug and excipients (Figure 2) \\
\hline Differential Scanning Calorimetry (DSC) & - & $\begin{array}{l}\text { No significant change in crystallinity of the drug; the } \\
\text { increase in dissolution rate is thus attributed to an increase } \\
\text { in available surface area of the nanoparticles (Figure 3) }\end{array}$ \\
\hline $\mathrm{X}$ - ray diffractometric analysis (XRD) & - & $\begin{array}{l}\text { Nanoprecipitation method does not change the crystalline } \\
\text { nature of drug which is in contrast to literature (Figure 4) }\end{array}$ \\
\hline Environment scanning electron microscopy (ESEM) & $\begin{array}{c}\text { After nanoprecipitation rod shaped crystals of the drug } \\
\text { converted to spherical in shape (Figure } 6 \text { ) }\end{array}$ & - \\
\hline
\end{tabular}

\#Where S.D is calculated for three experiments $(n=3)$

Table 2: Characterization of nanosuspensions. 
Citation: Pawar RN, Chavan SN, Menon MD (2016) Development, Characterization and Evaluation of Tinidazole Nanosuspension for Treatment of Amoebiasis. J Nanomed Nanotechnol 7: 413. doi: 10.4172/2157-7439.1000413

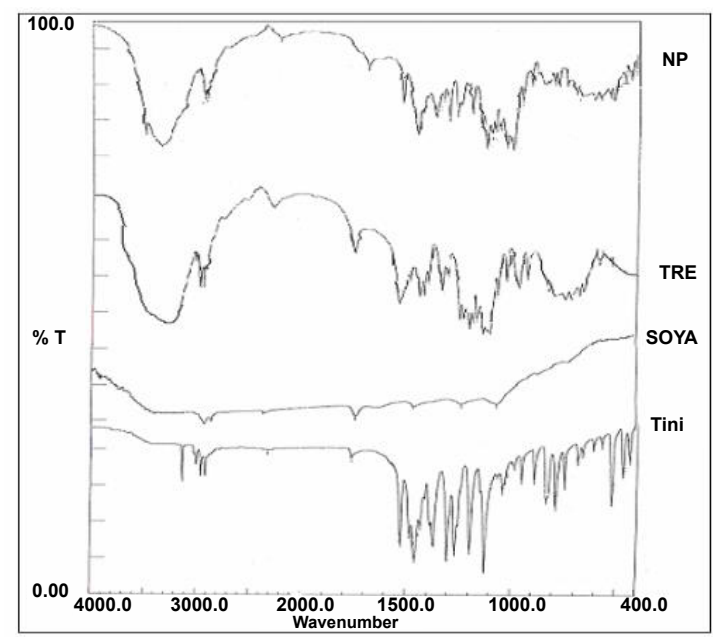

Figure 3: Comparative IR spectra of Tini (micronized Tinidazole), SOYA (soyalecithin), TRE (trehalose), NP (nanosuspension).

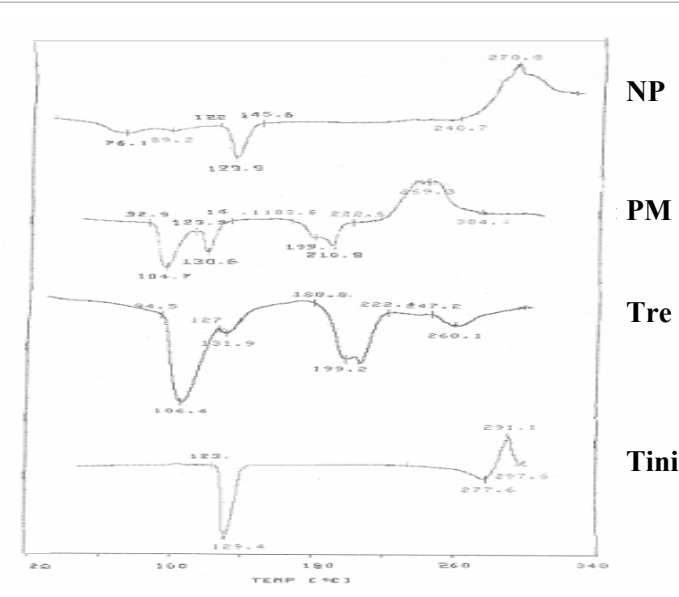

Figure 4: Comparative DSC of Tini (micronized Tinidazole), Tre (trehalose), PM (physical mixture), NP (nanoprecipitation sample).

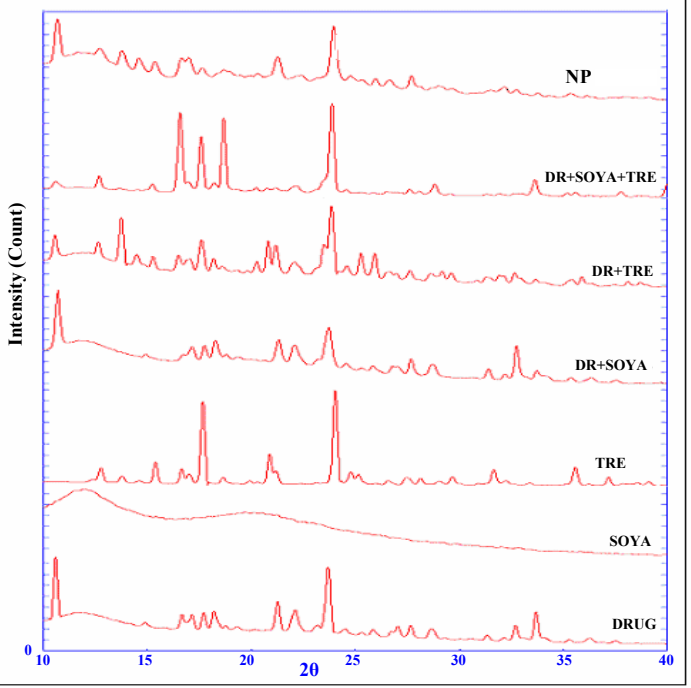

Figure 5: X-ray diffraction (XRD) DR (micronized Tinidazole), TRE (trehalose), SOYA (soyalecithin), NP (Nanosuspension).

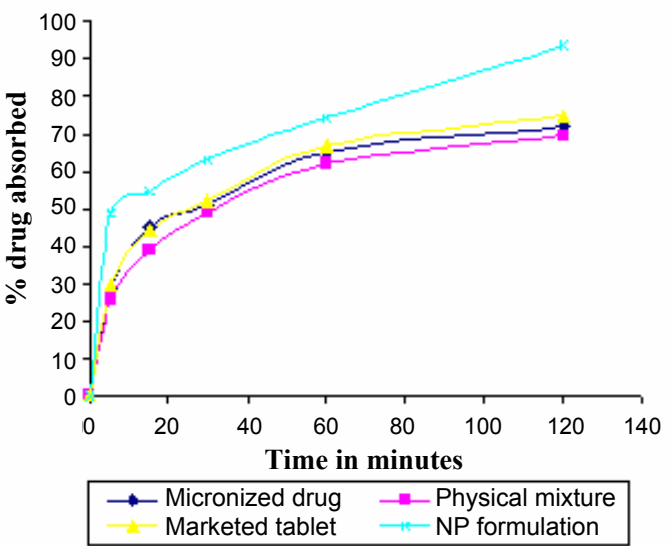

Figure 6: Ex- vivo absorption of drug through rat intestine.

a) Micronized TNZ

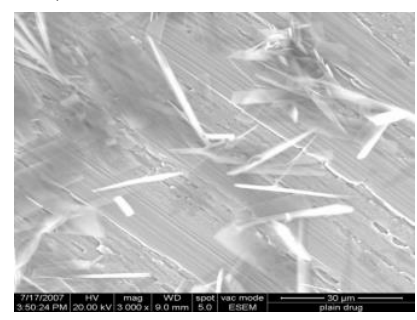

Figure 7: ESEM photograph. b) TNZ nanosuspension

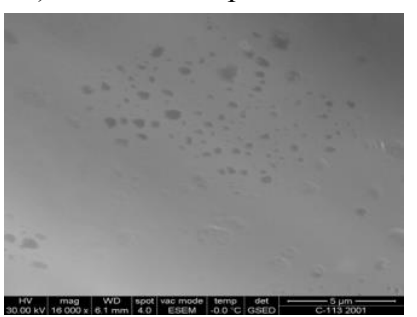

compared to micronized drug (72\%), physical mixture (70\%) and marketed tablet (75\%) within $2 \mathrm{hrs,} \mathrm{indicating} \mathrm{enhanced} \mathrm{bioavailability}$ from the nanosuspension product (Figures 1-7).

API-Micronized drug, PM NP-Physical mixture nanoprecipitation method, MKT TAB-Marketed tablet and NP-nanosuspension.

\section{Conclusion}

In conclusion, the present study has led to successful development of a stable, palatable nanosuspension of Tinidazole, with enhanced absorption, which would be suitable for pediatric patients. Differential Scanning Calorimetry (DSC) confirmed that the increase in dissolution rate is attributed to an increase in available surface area of the nanoparticles and conversion of rod shaped crystals of the drug to spherical shape confirmed by Environment scanning electron microscopy (ESEM).

\section{Acknowledgments}

The author is thankful to USV Pvt. Ltd. Govandi, Mumbai for providing freeze dryer facility.

\section{References}

1. Kocbek P, Baumgartner S, Kristl J (2006) Preparation and evaluation of nanosuspensions for enhancing the dissolution of poorly soluble drugs. International Journal of Pharmaceutics 312: 179-186.

2. Patravale VB, Date AA, Kulkarni RM (2004) Nanosuspensions: A promisingdrug delivery strategy. Journal of Pharmacy and Pharmacology 56: 827-840.

3. Gupta RB (2006) Fundamentals of Drug Nanoparticles. Nanoparticle technology for drug delivery Taylor and Francis, New York, USA.

4. Priya PV, Saritha BA, Shravani T (2016) Formulation and evaluation of irbesartan nanosuspension by precipitation method. Der Pharmacia Lettre 8 : 502-510. 
Citation: Pawar RN, Chavan SN, Menon MD (2016) Development, Characterization and Evaluation of Tinidazole Nanosuspension for Treatment of Amoebiasis. J Nanomed Nanotechnol 7: 413. doi: 10.4172/2157-7439.1000413

5. Shinde S, Hosmani A (2014) Preparation and evaluation of nanosuspensions for enhancing the dissolution of lornoxicam by antisolvent precipitation technique. Indo American Journal of Pharmaceutical Research 4: 398-405.

6. Kocbek P, Baumgartner S, Kristl J (2006) Preparation and evaluation of nanosuspensions for enhancing the dissolution of poorly soluble drugs. International Journal of Pharmaceutics 312: 179-186.

7. Chen Y, Liu J, Yang X, Zhao X, Xu H (2005) Oleanolic acid nanosuspensions: preparation , in-vitro characterization and enhanced hepatoprotective effect. Journal of Pharmacy and Pharmacology 57: 259-264.

8. Brough RO, Williams (2013) Amorphous solid dispersions and nano-crysta technologies for poorly water-soluble drug delivery. International journal of pharmaceutics 453: 157-66.

9. Borhade V, Pathak S, Sharma S, Patravale V (2014) Formulation and characterization of atovaquone nanosuspension for improved oral delivery in the treatment of malaria. Nanomedicine 9: 649-66

10. Merisko-Liversidge E, Liversidge GG (2011) Nanosizing for oral and parenteral drug delivery: a perspective on formulating poorly-water soluble compounds using wet media milling technology. Advanced drug delivery reviews 63: 427-40.
11. Kassem MA, Rahman AAA, Ghorab MM, Ahmed MB, et al. (2007) Nanosuspension as an ophthalmic delivery system for certain glucocorticoid drugs. International journal of pharmaceutics 340: 126-33.

12. Patel GV, Patel VB, Pathak A, Rajput SJ (2013) Nanosuspension of efavirenz for improved oral bioavailability: formulation optimization, in vitro, in situ and in vivo evaluation. Drug development and industrial pharmacy 40: 80-91.

13. Yadollahi R, Vasilev K, Simovic S (2015) Nanosuspension technologies for delivery of poorly soluble drugs. Journal of Nanomaterials.

14. Patel VR, Agrawal YK (2011) Nanosuspension: An approach to enhance solubility of drugs. Journal of advanced pharmaceutical technology and research 2: 81

15. Sudhakar B, NagaJyothi K, Murthy KVR (2014) Nanosuspensions as a versatile carrier based drug delivery systems-an overview. Current drug delivery 11: 299-305.

16. Wang Y, Zheng Y, Zhang L, Wang Q, Zhang D (2013) Stability of nanosuspensions in drug delivery. Journal of Controlled Release 172: 1126-41. 